\title{
An Enhanced Support Vector Regression Model for Weather Forecasting
}

\author{
R. Usha Rani ${ }^{1}$, Dr. T.K Rama Krishna Rao ${ }^{2}$ \\ Research Scholar, Dept of Computer science, Krishna University, Machilipatnam. \\ Andra Pradesh. INDIA-532201 \\ Professor, Dept of Information Technology \\ Aditya Institute of Technology and Management, Srikakulam, Andra Pradesh. INDIA-532201
}

\begin{abstract}
An attempt is made in this paper to develop an Enhanced Support Vector Regression (ESVR ) model with more un-interpretable kernel functions in the domain of forecasting the weather conditions. Every predicate model takes input data set parameters, processing with in specified levels of classification into variable sets and countered with a variable set reduction to reach the decision of prediction in a more confirm levels. This paper also provides a critical modeling design using the non linear regression namely support vector machines which is very non interpretable neural network designed as a two stage activity in prediction with more than three kernel functions for improving the performance of the SVMs experts in predicting the future weather happenings. Recording the input data set parameters of weather like temperature, water vapor, atmospheric pressure, dew point, wind speed, wind direction, rainfall, etc. compared with a MLP (Multi Layer preceptron) classification, SVM got much more esteemed performance in forecasting any one parameter with respect to others in a two stage procedure initial with self organizing maps and with best practice of more kernel functions investigation in weather forecasting.

Key Words: SVM, SOM, Kernel Functions, Classification and prediction, MLP.
\end{abstract}

\section{Introduction}

Weather conditions changes rapidly, weather forecast is a vital process, weather forecasting is a process of collecting data on atmospheric conditions, which records the temperature, humidity, rainfall, wind speed and its direction etc. high speed computers, wired and wireless sensors, meteorological satellites and weather radars are the tools used to collect the weather data for weather forecasting. Weather prediction is a complex process and a challenging task for researchers. It includes expertise in multiple disciplines [9]. The prediction of atmospheric parameters is essential for various applications.[3] Some of them include climate monitoring, drought detection, severe weather prediction, agriculture and production, planning in energy industry, aviation industry, communication, pollution dispersal etc. Accurate prediction of weather parameters is a difficult task due to the dynamic nature of atmosphere. Various techniques like linear regression, auto regression, Multi Layer Perceptron, Radial Basis Function networks are applied to predict atmospheric parameters like temperature, wind speed, rainfall, meteorological pollution etc. It was found that the non linear operator equations governing the atmospheric system are the ones who can better understand the dynamics of atmosphere. In the recent past many forecast methods have been developed using Artificial Neural Networks.[11,12, 7, 13].

Many meteorological departments are working on future weather prediction in order to prevent from the climatic hazardous. These department records the weather data represents in the form of images. The data recorded by the satellite represented in the form of images are made available to the public and the image processing with color, shape and textual information retrieval and content retrieval. Weather is no longer the unpredictable business variable. With advanced, powerful technology, one can anticipate changes in weather and keep one step ahead of Mother Nature rescuing.

\section{Multi Layer Preceptron}

In recent years, automatic indexing and retrieval based on image content has become more desirable for developing large volume image retrieval applications [8]. A model named "A Weather Forecasting System using concept of Soft Computing: A new approach" constructs an image, which represents the actual data. They relate those data to the forthcoming weather events based on their previous records and history or whatever recognized by their system [17].

The trained MLP of Artificial Neural Network can be effectively utilized for the prediction and classification of thunderstorm with appreciable level of accuracy. Its work reports the Artificial Neural Network design with minimum set of input parameter; however increase in input parameter will effectively increase the prediction accuracy[15],[1]. There are two rainfall prediction models which were developed and implemented in Alexandria, Egypt. These models are Artificial Neural Network ANN model and Multi Regression MLR 
model.[13],[14]. A Feed Forward Neural Network FFNN model was developed and implemented to predict the rainfall on yearly and monthly basis. A feed forward neural network with back propagation algorithm was implemented and tested for the purpose of yearly basis rainfall forecasting.

\section{Support Vector Machine}

Support Vector Machine algorithm relies on statistical learning theory. The principle of Support Vector Machines is to map the original data $\mathrm{X}$ into a feature space $\mathrm{F}$ with high dimensionality through a non linear mapping function and construct an optimal hyper plane in new space [3]. SVM techniques may be applied to both classification and regression. In classification, an optimal hyper plane is found that separates the data into two classes, whereas for regression a hyper plane is to be constructed that lies close to as many points as possible [5].

$$
G=\{(x t, d t)\} N_{1}^{t} .
$$

Support Vector Regression (SVR) predicts the maximum temperature at a location. Regression is the problem of estimating a function based on a given data set. [4] Consider a data set where $x i$ is the input vector, $d_{i}$ is the desired result and $N$ corresponds to the size of the data set. The general form of Support Vector Regression estimating function is $\mathrm{f}(x)=(w \varnothing(x))+b$ where $w$ and $b$ are the co-efficient that have to be estimated from data, where $\mathrm{f}(x)$ is the non linear function in feature space.[10]

The weather data is retrieved online through the weather reporting center like RMC (Regional Meteorological center) Chennai, for a year and is used to build the models. The database includes the data of several weather parameters recorded at every hour interval. The daily maximum temperature is extracted from the web service and is used for this work. The real world databases are prone to noisy and missing data [6].The data extracted from the service is preprocessed to improve the quality of data and thereby it improves the prediction results. In this research work, the maximum temperature of a day is predicted based on the maximum temperature of previous $n$ days where $n$ is the optimal length of the span [11]. The value of $n$ is found by experimental iterations. The work is implemented with selective attributes and scatter plots of SVM models are implemented with WEKA and Orange canvas along python2.7 scripting. The work has been taken the standard numerical processing values as measuring parameters given in "Estimation of precipitation during the period of south west monsoon using numerical [16].

\section{Methodology Used}

Step-wise contribution of non interpretable design given:

1. The dataset with respective parameters were framed.

2. The raw data to the tool and excel the values of all attributes, subsequently select attributes were loaded.

3. The query system called SVM classification expert along with self organizing map hierarchal tree designed with the kernel functions were investigated at process.

4. The output found by tree structured SOM to that of SVM were analyzed.

5. The current weather and predicate the future occurring conditions of weather were reported.

6. Accuracy of prediction is improvised through sending these resulted values again with self organizing Maps for self counter critic our result for more efficient prediction.

The key work is to have kernels functions investigations determining unknowing internal plane separation of class category happen.[1] More kernel functions validate our data output result in more refined and defined categorization takes place. The kernels could be linear, polynomial, rbf, or sigmoidal with efficient value of G,C,D.

Parameters of SVM: Kernel Parameters, degree of polynomial, additive constant, Error weight in training error and check out through the Cost-SVM, complexity bound-SVM.

The weather parameters input are:

svm. weather $\leftarrow \operatorname{svm}$ (data $=$ "50 profiles",

labels = "by an SVM expert",

kernel $=$ "..",

parameters $=" . . ")$

svm.reportanalysis $\leftarrow$ predict(svm.weather,new.weather_reportanalysis).

It's very non-interpretable model of predicting the weather happ

enings through framing of dataset with typical changes in defined dataset. This is a simple mining procedure with lot more thousands of observations gained knowledge tries to provide a statistical approach in classifying the different weather conditions and promote to [2] explore a decision making system with the hierarchal clustering technique .Materializing the numerical weather prediction can be headed through image sensing in the next work progress. The current study is based on the literature study about the classification and predication 
methods on weather reporting by neural network data training. The effectiveness of predicate variable in kernel trick is revised and the resulted efficiency of MLP, single SVM and with hierarchal tree SOM is shown in below charted figure1.

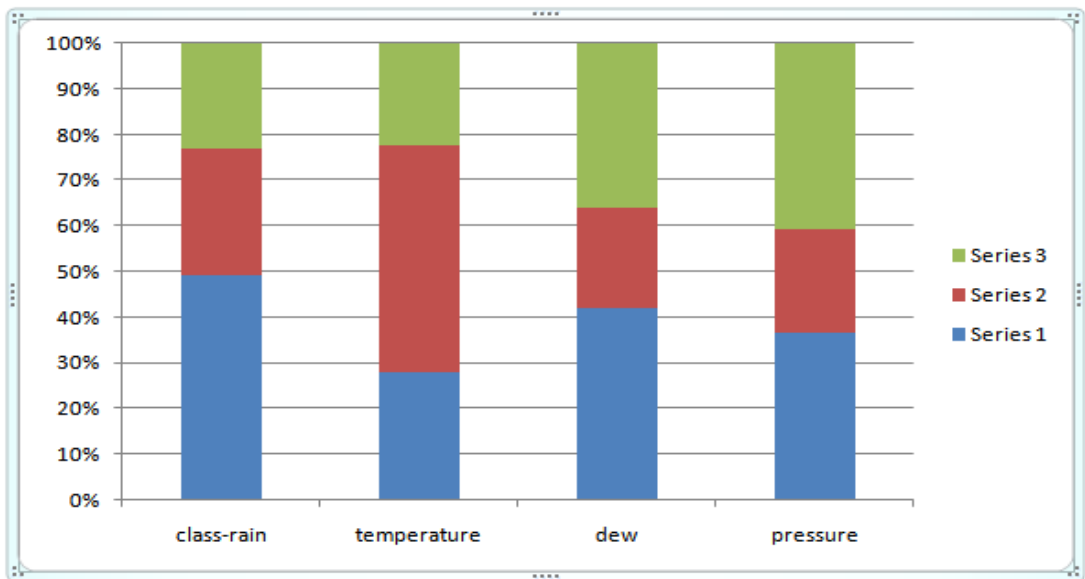

Fig:1 percentage of classification through the MLP,SVM single, and SVM with SOM in given parameters.

The multilayer perceptron which resulted in classify the categories of conditions of weather namely sunny, rainy, cloudy, foggy based on the variables or attributes the attributes like wind direction, humidity, temperature, rainfall, speed of the wind, precipitation etc, with the revised knowledge work enhancement of tricky kernel functions and their more number in investigation provide us path of efficiency proceeded along with SVM and SOM.In the result graph the Series 1,Series 2, Series 3 are the MLP, SVM with SOM, and SVMSingle based analysis report on effectivenes in process of prediction. The SVM with SOM good estimated quantitative prediction given.

\section{Experimental Results and Analysis}

We have many modeling algorithms namely linear regression, cluster analysis, multiple linear regression, k-means regression, artificial neural networks and principal component analysis. In whole of modeling we go with support vector machines. They define a kernel and an exponential term that frames a class label and do categorical classification. Report the current weather and predicate the future occurring conditions of weather. Accuracy of prediction is improvised through sending these resulted values again with self organizing Maps for self counter critic our result for more efficient prediction.

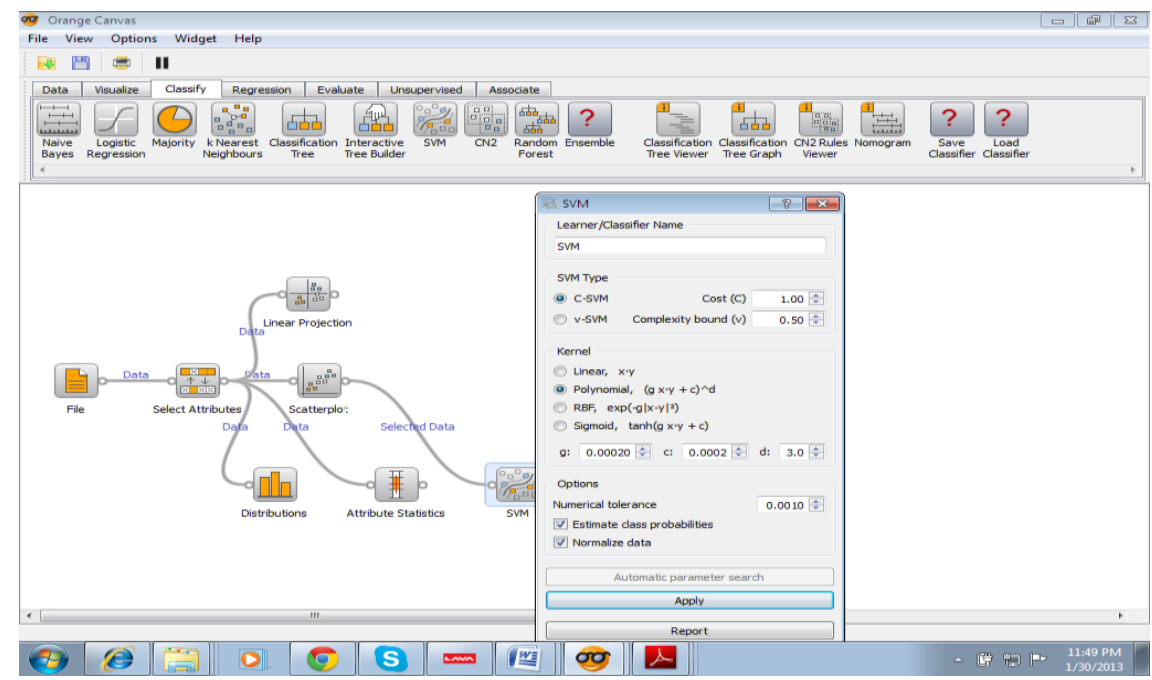

Fig:2 Orange Canvas with SVM’s work.

\section{Conclusion And Future Work}

This design predicate model of neural networks provides us the more kernel functions hybrid procedure for classification and prediction using MLP for initial classification that followed by the single SVM and SVM with SOM. SOMs are used to classify a given input into one of the partitioned regions based on a tree-structured architecture and considering SVMs expert is used to produce the output. In this implementation model we come 
up with "hard" decision used in the current partition, smoothly evaluate with the refined outliers utilization. This result improvised more number of kernel functions is the one way of indexing the efficiency of the model designed and the future path of work can be compared with different variable set reduction modeling approaches and work with effective content retrieval images and hurricanes data.

\section{References}

[1]. Tk.Rama Krishna Rao, R,Usha Rani Content Based Image Retrieval Through Feed Forward Neural Networks 2012 IEEE 8th International Colloquium on Signal Processing and its Applications, Malek, Malaysia 23-25 March.

[2]. Classification and Prediction of Future Weather by using Back Propagation Algorithm-An Approach. Sanjay D. Sawaitul1, Prof. K. P. Wagh2, Dr. P. N. Chatur3, www.ijetae.com (ISSN 2250-2459, Volume 2, Issue 1, January 2012)

[3]. Amit Jain, B. Satish, Clustering Based Short TermLoad Forecasting using Support Vector Machines, 2009 IEEE Bucharest Power Tech Conference, June 28th -July 2nd, Bucharest, Romania.

[4]. Y.Radhika and M.Shashi, Atmospheric Temperature Prediction using Support Vector Machines, International Journal of Computer Theory and Engineering, Vol. 1, No.1, April 2009, 1793-8201.

[5]. Amit Jain, B. Satish, Integrated Approach for ShortTerm Load Forecasting using SVM and ANN, TENCON 2008. IEEE Region 10 Conference 19-21 Nov. 2008 Page(s): 1 - 6 Digital Object Identifier

[6]. Guhathakurtha, P.,"Long-Range monsoon rainfall prediction of 2005 for the districts and sub-division kerala with artificial neural network."Current Science, Vol.90, No.6,25. 2006

[7]. Lijuan Cao. Support vector machines experts for time series forecasting. www.elsevier.com/locate/neucom, Neurocomputing 51 (2003) $321-339$.

[8]. Tung A.K.H., "Efficient mining of intertransaction associationrules", IEEE Trans. on Knowledge and Data Engineering,vol.15(1), 43-56p, Jan./ Feb. 2003.

[9]. Denis Riordan, and Bjarne K Hansen, “A fuzzy case-based system for weather prediction.” Engineering Intelligent Systems, Vol .10, No.3. 2002 .

[10]. Ronan Collobert and Samy Benegio, SVM Torch: Support Vector Machines for Large-Scale Regression Problems, Journal of Machine Learning Research, 1, pp.143- 160. 2001,3] Haykin, S., "Neural Networks- A Comprehensive Foundation."Prentice Hall. 1999.

[11]. K.R. Muller, A.J. Smola, G. Ratsch, B. Scholkopf, J. Kohlmorgen, Using support vector machines for time series prediction, in: B. Scholkopf, C.J.C. Burges, A.J. Smola (Eds.), Advances in Kernel Methods-Support Vector Learning, MIT Press, Cambridge, MA, 1999, pp. 243-254.

[12]. S. Mukherjee, E. Osuna, F. Girosi, Nonlinear prediction of chaotic time series using support vector machines, in: NNSP'97: Neural Networks for Signal Processing VII: Proceedings of the IEEE Signal Processing Society Workshop, 1997, Amelia Island, FL, USA, pp. 511-520.

[13]. K.R. Muller, J.A. Smola, G. Ratsch, B. Scholkopf, J. Kohlmorgen, V.N. Vapnik, Predicting time series with support vector machines, in: ICANN'97: Proceedings ofthe sevnth International Conference on Arti3cial Neural Networks, Lausanne, Switzerland, 1997, pp. 999-1004.

[14]. J. Nie, Nonlinear time series forecasting: a fuzzy-neural approach, Neurocomputing (1997) 63-76. Neurocomputing, Volume 16, Number 1, 1 July 1997 , pp. 63-76.Elsiever.

[15]. Sharma A.,"A Weather Forecasting System using concept of Soft Computing: A new approach”2006, IEEE Advanced computing and communications.

[16]. R.Usha Rani, T.K.Rama Krishna Rao."Estimation of precipitation during the period of south west monsoon using numerical methods under C programming,IJCET, Vol-4,Issue 3,May-June 2013. 